\title{
Optimization of Recloser Placement to Improve Reliability by Genetic Algorithm
}

\author{
Nematollah Dehghani ${ }^{1}$, Rahman Dashti ${ }^{2}$ \\ ${ }^{1}$ Young Researchers Club of Islamic Azad University, Bushehr Branch, Bushehr, Iran \\ ${ }^{2}$ Department of Electrical and Computer Engineering, Islamic Azad University, Bushehr Branch, Bushehr, Iran \\ E-mail:nemat_dehghani@yahoo.com,rdashti@yahoo.com \\ Received April 23, 2011; revised May 27, 2011; accepted June 11, 2011
}

\begin{abstract}
In this paper, a simple method for placing an optimal number of recloser is presented. The algorithm is solved using genetic algorithm as the optimization method. The majority of outage events experienced by customers are due to electrical distribution failures. Increasing network reliability is a necessity in order to reduce interruption events. Distribution network automation can trim down outage events and increase system reliability. Network automation has to be done using optimization approaches. Genetic Algorithm (GA) is a relatively new technique used in power systems optimization problems. Distribution network automation is one of the aspects tackled using GA. However, the methodologies used to improve the reliability of radial distribution feeders are reviewed. The reliability improvement is demonstrated for typical distribution feeder layouts determined. The method enjoys the simplicity of configure uration, accuracy of the results and reduction of the time consuming. The obtained results also show the applicability of the algorithm.
\end{abstract}

Keywords: Recloser, Reliability, Transient Error, GA

\section{Introduction}

The necessity to provide reliability in the distribution system and in power can be defined as the system's capability in generation, transmission and distribution of electricity energy to the consumers. In the distribution part, halt frequent (frequency of occurrence of halts and interrupts), the time period of each halt and the rate of consumption each consumer needs in the lake of consumer service and lake of feeding system are the main factor s to judge about systems reliability.

Many factors are important in the determining the reliability of a system that some of them are controllable. These factors are depend on variable such as the reliability of the equipment components, networks length its loading, networks configuration loads characteristic.

Other factor are also important in reduction of network s reliability such as disorder and distribution by third part ,atmospheric and environmental condition like temperature, humidity ,environmental, and air pollution, wind, rain, rain, snow ,ice, and thunderbolt.

\section{Type of Errors in Power System}

Generally, errors created in the electricity system in the term of cause and time of the error continuation are two section inside and outside errors.

Considering the above, short circuit is derided to passive short circuit and permanent short circuit. Whereas about 80 percentage of error in medium voltage distribution system is passive and elimination after one or two time switching of station after automatic interrupts. That is a try and error performance to detect of the errors that causes sever stress and creation of special transient modes in network and also causes the reduction of station's key life time. so, in order to prevent unsuccessful switching from transmission and high distribution system and to increase from transmission and high distribution systems and to increase the reliability in transmission of electrical energy and establishing its continuity and also creating stability in system of medium voltage lines, a soft were installation called recloser is required that should meet following cases:

- No repeated switching of station in transient errors.

- No switching off of the loads because of lines problem in one region.

- Considering the above, the place of recloser's installation and the aims witch the place of installation is determined has specific importance. There are two views in the positioning of recloser system.

- Recloser's positioning from the view of cost's reduc- 
tion.

- Recloseer's positioning in order to reduce transient interruption and reduction of undistributed energy and satisfaction of customers.

This report deals with second view of recloser positioning that is reduction of transient interruptions and thus reduction in undistributed energy and satisfaction of customers.

This paper concern with the collection of total average frequency of occurred errors in the region.

\section{Genetic Algorithm}

A Genetic algorithm (GA) is an optimization based on the mechanics of natural selection and natural genetic. GA is inspired by natural genetics and a Darwinian theory of evolution. A genetic algorithm involves simulating competition between a numbers of individuals who represent solution to problem. A set of genes which corresponds to a "chromosome" in natural genetic is referred to as "string" in a GA [1,2]. The mechanics of GAs are surprisingly simple, involving nothing more complex than copying string and swapping partial string. GAs start with a population of string and thereafter, generate successive population using the following three basic operator generation, crossover, mutation [3]. Concisely stated, a genetic algorithm is a programming technique that mimics biological evolution as a problem-solving strategy. Given a specific problem to solve, the input to the GA is a set of potential solutions to that problem, encoded in some fashion, and a metric called a fitness function that allows each candidate to be quantitatively evaluated [4]. These candidates may be solutions already known to work, with the aim of the GA being to improve them, but more often they are generated at random. The GA then evaluates each candidate according to the fitness function. In a pool of randomly generated candidates, of course, most will not work at all, and these will be deleted. However, purely by chance, a few may hold promise - they may show activity, even if only weak and imperfect activity, toward solving the problem. These promising candidates are kept and allowed to reproduce. Multiple copies are made of them, but the copies are not perfect; random changes are introduced during the copying process. These digital offspring then go on to the next generation, forming a new pool of candidate solutions, and are subjected to a second round of fitness evaluation. Those candidate solutions which were worsened, or made no better, by the changes to their code are again deleted; but again, purely by chance, the random variations introduced into the population may have improved some individuals, making them into better, more complete or more efficient solutions to the problem at hand. Again these winning individuals are selected and copied over into the next generation with random changes, and the process repeats. The expectation is that the average fitness of the population will increase each round, and so by repeating this process for hundreds or thousands of rounds, very good solutions to the problem can be discovered. As astonishing and counterintuitive as it may seem to some, genetic algorithms have proven to be an enormously powerful and successful problem-solving strategy, dramatically demonstrating the power of evolutionary principles. Genetic algorithms have been used in a wide variety of fields to evolve solutions to problems as difficult as or more difficult than those faced by human designers [5]. Moreover, the solutions they come up with are often more efficient, more elegant, or more complex than anything comparable a human engineer would produce. In some cases, genetic algorithms have come up with solutions that baffle the programmers who wrote the algorithms in the first place!

\section{Reliability Characters}

In a conventional radial feeder, reclosers are only expected to detect the unidirectional flow of current. Typically, a recloser upstream from the fault location detects the fault current, trips, and goes into a predefined reclosing sequence in order to restore service, if the fault is of a temporary nature. If more reclosers are present on the radial feeder, they are time coordinated, usually using Inverse Definite Minimum Time (IDMT) curves [6]. IDMT allows for the recloser operating time to be inversely proportional to the magnitude of the fault current, forcing the recloser closest to the fault to operate first and clear the fault.

The placement of protection devices in a conventional (radial) feeder is designed to maximize network reliability, and therefore minimize the traditional reliability indices assuming that the energy source is located only at the substation. Typically, utilities use standardized indices such as SAIFI and SAIDI, which measure the average accumulated duration and frequency of sustained interruptions per customer $[7,8]$. The system average interruption frequency index (SAIFI) and the system average interruption duration index (SAIDI) are defined as follows:

$$
\begin{aligned}
& S A I F I=\frac{\sum \lambda_{i} \cdot N_{i}}{N_{T}} \\
& S A I D I=\frac{\sum r_{i} \cdot N_{i}}{N_{T}}
\end{aligned}
$$

where $N_{i}$ is the number of interrupted customers for each 
interruption event, $N_{T}$ total number of customers, and $r_{i}$ is the restoration time for each interruption event. Customer average interruption duration index (CAIDI) is defined as the average time required to restore service to the average customer per sustained interruption:

$$
C A I D I=\frac{\sum r_{i} \cdot N_{i}}{\sum \lambda_{i} \cdot N_{i}}=\frac{S A I D I}{S A I F I}
$$

The average service availability index $(A S A I)$, represents the fraction of time that a customer has power provided during one year (or other defined reporting period). Assuming one year period (8760 hours), it is calculated via:

$$
A S A I=\frac{8760-S A I D I}{8760}
$$

As the importance of temporary faults increases, more utilities are starting to track them using the MAIFIe index, which measures the number of momentary interruptions per customer.

The momentary average interruption event frequency index (MAIFIe) is defined

$$
\text { MAIFIe }=\frac{\sum I D_{i} \cdot N_{i}}{N_{T}}
$$

where $I D_{i}$ is the number of interrupting device operations.

The recloser placement can be optimized with respect to any of these, or some other, indices. To include the effects of both sustained and momentary interruptions, a composite index may be used, as defined below.

$$
\left[\begin{array}{l}
C=W_{1} \frac{S A I F I-S A I F I_{T}}{S A I F I_{T}}+W_{2} \frac{S A I D I-S A I D I_{T}}{S A I D I_{T}} \\
+W_{3} \frac{M A I F I e-\text { MAIFIe }_{T}}{\text { MAIFIe }_{T}}
\end{array}\right]
$$

where $W_{1}, W_{2}$ and $W_{3}$ are weights for indices SAIFI, $S A I D I$, and MAIFIe, respectively, and the subscript $T$ indicates the target value [9].

The percentage of different companies imply different variables of reliability shown in Figure 1 [10].

\section{Problem Definition Using}

In this stage after importing system's information $\mathrm{n}$ bit of 0,1 is considered equal to the number of lines candidate for recloser (Figure 2).

Genetic algorithm concerns with generation of different gene. Calculations are based on a system of genes generation. If the $I$ bit value of 1 (that is between $I$ and $i-1$ line would be without recloser and has no effect on calculation. It should be noted that found to be not suitable for recloser by experience, could be locked perma-

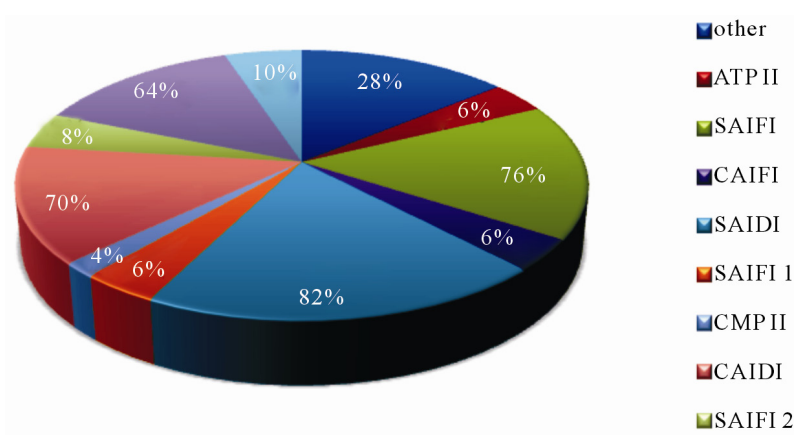

Figure 1. Percentage of companies using indices (49 companies responded).

nently though genetics toolbox like the most of terminal shins.

\section{The Effects of Network Simulation Result on the Standard System in Order to Checking Variables}

For testing the program, first it should be evaluated by the standard testing system (RBTS) in Figure 3.

The information is shown in Tables $\mathbf{1}$ and $\mathbf{2}$ and the result is shown in Table 3.

\section{Simulation Result on the Standard Power System}

Reference number [5] suggests placing a recloser at the half-way point of the radial feeder, assuming uniformly distributed load. This would yield a 50\% reliability improvement to customers upstream from the recloser. Similarly, locations at $1 / 3$ and $2 / 3$ of feeder length should be considered if two reclosers are to be placed. In

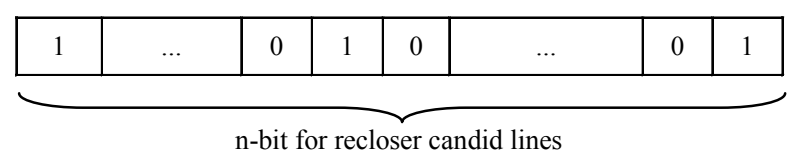

Figure 2. Coding of variable. $N$ : the number of communicative lines between shins.

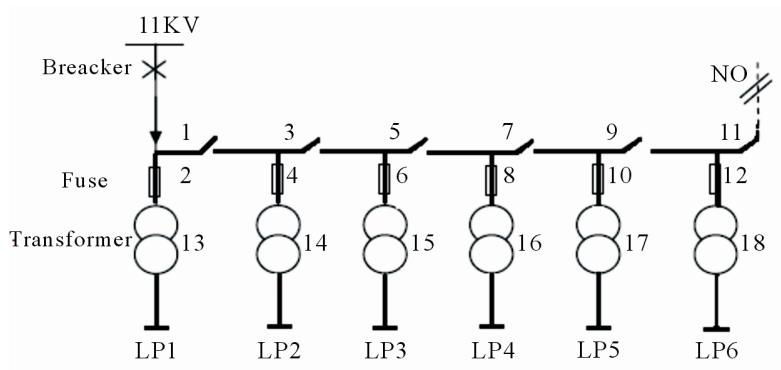

Figure 3. Standard test power system (rbts). Lp: the poin of load (load point). 
Table 1. Data of estandard feeder-RBTS-ODD SECTIONS.

\begin{tabular}{ccccc}
\hline Main section & Length (km) & $\lambda(\mathbf{f} / \mathbf{y r})$ & $r(\mathrm{hr} / \mathrm{f})$ & $S(\mathrm{hr} / \mathrm{f})$ \\
\hline 1 & 0.75 & 0.04875 & 5 & 1 \\
3 & 0.60 & 0.03900 & 5 & 1 \\
5 & 0.75 & 0.04875 & 5 & 1 \\
7 & 0.75 & 0.04875 & 5 & 11 \\
9 & 0.60 & 0.03900 & 5 & 1 \\
11 & 0.80 & 0.05200 & 5 & 1 \\
\hline
\end{tabular}

Table 2. data of standard feeder-RBTS-EVEN SECTIONS.

\begin{tabular}{|c|c|c|c|c|c|c|c|}
\hline $\begin{array}{l}\text { Lateral } \\
\text { section }\end{array}$ & $\begin{array}{l}\text { Length } \\
(\mathbf{k m})\end{array}$ & $\begin{array}{c}\lambda \\
(\mathbf{f} / \mathbf{y r})\end{array}$ & $R(\mathbf{h r} / \mathbf{f})$ & $\begin{array}{c}S \\
(\mathbf{h r} / \mathbf{f})\end{array}$ & Transformer & $\begin{array}{c}\lambda \\
(\mathbf{f} / \mathbf{y})\end{array}$ & $\begin{array}{c}r l \\
(\mathbf{h r} / \mathbf{f})\end{array}$ \\
\hline 2 & 0.60 & 0.03900 & 5 & 1 & 13 & 0.015 & 10 \\
\hline 4 & 0.80 & 0.05200 & 5 & 1 & 14 & 0.015 & 10 \\
\hline 6 & 0.75 & 0.04875 & 5 & 1 & 15 & 0.015 & 10 \\
\hline 8 & 0.60 & 0.03900 & 5 & 1 & 16 & 0.015 & 10 \\
\hline 10 & 0.75 & 0.04875 & 5 & 1 & 17 & 0.015 & 10 \\
\hline 12 & 0.65 & 0.03900 & 5 & 1 & 18 & 0.015 & 10 \\
\hline
\end{tabular}

real life situations, which include the presence of critical loads and non-uniform load distributions, utilities often resort to engineering judgment to place reclosers according to the reliability guidelines.

In this paper we using genetic algorithm to find best position for recloser. This is important step using GA for optimal allocation recloser in power system with 70 bus Figure 4.

This part is concern with optimal positioning of recloser by the method that is used in the system of Figure 4 and its result are compared with reference [5], Table 3 shows comparing result.

\section{Final Simulation Result}

All simulation is done in MATLAB software environment. The number of population member and the number of generation is chosen for solving the question.

Crossover probability is set to 0.8 mutation probability is set as following At first, completing algorithm starts from 0.1 and then reduces in each generation and at last gets to 0.001 (Table 4).

It could be seen that reliability is increased in large amount.

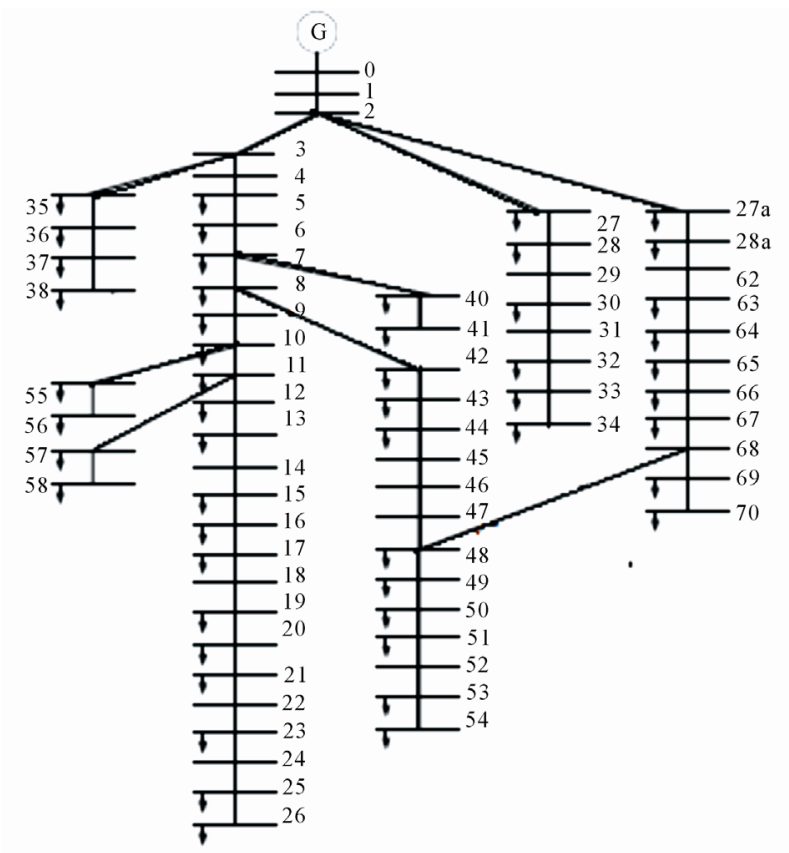

Figure 4. 70-bus test power system.

Table 3. Comparing result.

\begin{tabular}{ccc}
\hline $\begin{array}{c}\text { Result in } \\
\text { reference [5] }\end{array}$ & $\begin{array}{c}\text { Result in } \\
\text { objective function } \\
\text { by GA }\end{array}$ & $\begin{array}{c}\text { Reliability } \\
\text { characters }\end{array}$ \\
\hline 0.3355 & 0.3355 & SAIFI \\
0.8328 & 0.8328 & SAIDI \\
\hline
\end{tabular}

Table 4. Final simulation result by GA.

\begin{tabular}{cccc}
\hline $\begin{array}{c}\text { Position of } \\
\text { recloser }\end{array}$ & $\begin{array}{c}\text { Final result in } \\
\text { refrence [5] }\end{array}$ & $\begin{array}{c}\text { Final result } \\
\text { in objective } \\
\text { function by } \\
\text { GA }\end{array}$ & $\begin{array}{c}\text { Number of } \\
\text { recloser }\end{array}$ \\
\hline $8-9$ & 3.9560 & 3.5424 & 1 \\
$8-9$, & 2.8695 & 2.0847 & 2 \\
$30-31$ & & & \\
$3-4,30-$ & 1.9012 & 1.1937 & 3 \\
$31,47-48$ & & & \\
\hline
\end{tabular}

\section{Conclusions}

In this paper we study one of the protective device in electrical power system, this device can recognize of unusual states for example transient errors can do best activity, other than the passing fault have important rule in position and act of recloser.

This paper has presented an effective application of the genetic algorithm optimization to practical distribution system automation. One location may satisfy an objective function subject to a specific reliability index. However, adding reliability index as a constraint can 
result in different optimum locations. Multiple reliability indices were combined to form an objective function using the concept of a composite index in which the weights and target values of each index must be determined.

Genetic Algorithm optimization has been shown to be an effective technique to optimize the automation of some electrical distribution systems as has been illustrated.

This paper is studying the networks which encounter high time outage due to the transient faults. After presenting the GA for optimal allocating of recloser and it's simulation on a 70 buses network, we can improve reliability of system and after that we comparing result with reference [5] and find GA is best method to optimization .The results demonstrate the capability and applicability of the proposed method.

\section{Suggestion}

1) In addition to finding problematic lines and prioritization of them according to sensitive and important places, using and installing the recloser requires and important places, using and installing the recloser requires suitable installation place and correct setting with regard to recloser facilities, in order to have return on capital equal to customers satisfaction.

Otherwise, not only the customers satisfaction would not be achieved, but also, huge costs will be imposed on distribution company.

2) Installation and setting of recloser should be done by expert.

3) If using the recloser, their subsidiary equipment should be reviewed and mentioned in order.

Obtain more economic interests through optimal using of them.

4) These studies show that, to be more economic in using recloser, not only there installation and commis- sioning is important but also several studies should be done about feeders and their type of load to get acceptable results.

\section{References}

[1] D. E. Goldberg, "Genetic Algorithms in Search, Optimization and Machine Learning," Addison-Wesley, Longman, London, 1998

[2] O. Pavel, "Optimization of Parameters of Fuzzy Controllers by Genetic Algorithm," Technical University of Berno, Faculty of Mechanical Engineering, Borno, 2010.

[3] E. M. Rudinck, J. H. Patel, G. S. Greenstein and T. M. Niermann, "Sequential Circuit Test Generation in Genetic Algorithm Framework," Proceedings of ACM/IEEE Design Automation Conference, San Diego, 6-10 June 1994, pp. 698- 704.

[4] M. Kalanter, R. Dashti and R. Dashti, "Combination of Network. Recon Figuration and Capacitor Placement for Loss Reduction in Disterbution System with Based Genetic Algorithm," UPEC Conference, New Castle, 6-8 September 2006.

[5] Z. Feng, "Electric Distribution System Risk Assessment Using Actual Utility Reliability Data," Thesis, March 2006, pp. 20-24.

[6] A. R. Bergen, "Power System Analysis," 1st Edition, Prentice-Hall, Upper Saddle River, 1986.

[7] IEEE, "IEEE Trial-Use Guide for Electric Power Distribution Reliability Indices," IEEE 1366-1998 Standard, 1998

[8] C. A. Warren, R. Ammon and G. Welch, "A Survey of Distribution Reliability Measurement Practices in the US," IEEE Transactions on Power Delivery, Vol. 14, No. 1, 1999, pp. 250-257. doi:10.1109/61.736733

[9] A. Pregelj, "Impact of Distributed Generation on Power Network Operation," Ph.D. Thesis, School of Electrical and Computer Engineering, Georgia Institute of Technology, Atlanta, 2003.

[10] T. Guonen, S. M. Rezaei, "Distribution Engineering," Tehran University Publishing, Tehran, No. 1-1375 